\title{
The Neurorehabilitation Training Toolkit (NTT): A Novel Worldwide Accessible Motor Training Approach for At-Home Rehabilitation after Stroke
}

\author{
Sergi Bermúdez i Badia and M. S. Cameirão \\ Madeira Interactive Technologies Institute, Universidade da Madeira, Campus Universitario da Penteada, Madeira, \\ 9020-105 Funchal, Portugal \\ Correspondence should be addressed to Sergi Bermúdez i Badia, sergi.bermudez@m-iti.org
}

Received 16 November 2011; Revised 6 February 2012; Accepted 13 February 2012

Academic Editor: Agnès Roby-Brami

Copyright (C 2012 S. Bermúdez i Badia and M. S. Cameirão. This is an open access article distributed under the Creative Commons Attribution License, which permits unrestricted use, distribution, and reproduction in any medium, provided the original work is properly cited.

\begin{abstract}
After stroke, enduring rehabilitation is required for maximum recovery, and ideally throughout life to prevent functional deterioration. Hence we developed a new concept for at-home low-cost motor rehabilitation, the NTT, an Internet-based interactive system for upper-limb rehabilitation. In this paper we present the NTT design concepts, its implementation and a proof of concept study with 10 healthy participants. The NTT brings together concepts of optimal learning, engagement, and storytelling to deliver a personalized training to its users. In this study we evaluate the feasibility of NTT as a tool capable of automatically assessing and adapting to its user. This is achieved by means of a psychometric study where we show that the NTT is able to assess movement kinematics-movement smoothness, range of motion, arm displacement and arm coordinationin healthy users. Subsequently, a modeling approach is presented to understand how the measured movement kinematics relate to training parameters, and how these can be modified to adapt the training to meet the needs of patients. Finally, an adaptive algorithm for the personalization of training considering motivational and performance aspects is proposed. In the next phase we will deploy and evaluate the NTT with stroke patients at their homes.
\end{abstract}

\section{Introduction}

There are about 16 million new strokes per year worldwide [1], and about 5 million of the survivors will sustain motor and/or cognitive impairments for the rest of their lives [2]. This situation leads to high societal costs in medical care and rehabilitation expenses, with annual costs above $€ 38$ billion in Europe [3]. Collaterally, there is decreasing participation of these patients in professional and social life since stroke survivors frequently suffer from mood disorders or depression $[4,5]$. Therefore, due to its direct and indirect effects, stroke is one of the main contributors for the worldwide burden of disease $[6,7]$.

Following stroke, enduring rehabilitation is needed for maximum recovery. This requires long-term hospitalization or outpatient rehabilitation, a situation that is extremely demanding both for patients and national health systems. In fact, there is a growing interest towards early supported discharge from hospitals and at-home rehabilitation [8, 9]. Nonetheless, despite outpatient rehabilitation programs, it is generally assumed that the full potential for recovery is reached in the first 6 months after stroke, with patients then being discharged from rehabilitation [10]. This might be problematic as there is evidence that the brain remains plastic at later stages post stroke, meaning that there can still be place for additional recovery $[11,12]$. Ideally, stroke patients should undergo maintenance rehabilitation throughout life to prevent functional deterioration. Indeed, a significant decline in mobility after rehabilitation discharge is expected in one fifth of chronic patients, having a direct impact in performing activities of daily living (ADL) [13]. Thus, there is a need to find solutions to provide patients with tools that allow them to have enduring rehabilitation at their homes.

In recent years, novel technology-based systems have been developed for at-home stroke rehabilitation [14-23]. Although the price of these systems is lower than standard 
technology-based rehabilitation devices, most of them are still unaffordable for the majority of patients. Moreover, these systems usually rely on particular hardware components, require elaborate setups, and/or need remote guidance, which makes them difficult to use particularly if we take into account that most of the stroke patients are elderly.

To provide patients with effective, uncomplicated, and inexpensive rehabilitation at their homes, we have developed the Neurorehabilitation Training Toolkit (NTT), a PC-based interactive system for upper-limb rehabilitation. The NTT makes use of well-established state of the art requirements for effective rehabilitation after stroke, providing training that is frequent, reiterative, and task specific [24-26], and that presents feedback on performance and outcomes [2729]. These characteristics are achieved through the use of game-like tasks displayed on a standard computer, designed to address the specific upper-limb deficits of stroke patients.

In the last few years, a number of standard commercial videogames have been used for stroke rehabilitation [30, 31]. Although the concept is valid, these are ad hoc solutions since these games were designed for a different target population, therefore not fully addressing the needs and capabilities of the patients. In previous work we developed and evaluated a game for rehabilitation that provides personalized training that is adjusted to the individual capacities of the patients [32]. In this way, patients can undergo a challenging training task designed towards their specific motor deficits (thus avoiding boredom), without falling beyond the patients capabilities which could lead to frustration. We have shown that the use of such an approach as a complement to standard rehabilitation leads to improved and accelerated recovery in acute stroke patients [18]. With the NTT, we go one step further because we are able to assess movement kinematics and psychometrics that allow the personalization of a number of training objectives. These objectives include the range of motion and movement smoothness.

The NTT consists of widely available home-based technologies, namely, a PC or laptop, two mice, and an Internet connection. This configuration makes the system uncomplicated and rather inexpensive, and therefore accessible and affordable for most patients. The use of an Internet connection is twofold. On one hand, the game "lives" onlinefor an easy upgradeability of the software-and requires no installation. On the other hand, it can be used to monitor and assess the progress of patients remotely. Additionally, the NTT encompasses automatic questionnaires to assess the usability, engagement, and acceptance of the training tasks, as well as to measure the training impact on the performance of ADLs. Here we present the design concepts behind the NTT, the results of a pilot study where we developed a novel personalization algorithm based on functional motor outcomes, and demonstrate the NTT capabilities as an assessment and monitoring tool tested with healthy users. Due to its simplicity and innovative features, we believe that the NTT is a powerful tool that will give patients access to an affordable, effective, and long-term rehabilitation at their homes, allowing them to maximize and sustain recovery while increasing their overall quality of life.

\section{Materials and Methods}

The NTT is a software-based motor training toolkit that is accessed and executed from Internet as a web applet by means of an Internet browser (Figure 1(A)). NTT has been verified to work with the most commonly used browsers such as Internet Explorer, Google Chrome, Safari, Firefox, and Opera. In this context, the web browser serves as the interface to the training toolkit, allowing its execution without needing local installation or additional software besides the NTT applet itself. In addition to a PC with an Internet connection, the NTT user needs a working email account for communication and user support if needed.

On the hardware side, the NTT can run on any modern computer with Windows O.S. (Windows XP, Vista, or 7) (Figure $1(\mathrm{~B})$ ). The only requirement for the $\mathrm{PC}$ is to have two mice connected to it. Computer mice are widespread, reliable, and extremely low-cost devices that will serve to track the physical movements of the arms of the patients, which in turn will be used to interact with the NTT. Technically, the main advantage of the NTT is the ability to capitalize on existing common hardware to deliver personalized and continued training. This is achieved by the low hardware requirements and the accessibility of our online system (Figure $1(\mathrm{C})$ ). Internet serves as the distribution channel for the NTT, offering its training and other advantages to any patient anywhere in the world with access to a PC and an Internet connection. Furthermore, NTT upgrades are immediately deployed to the user's home without requiring action from their side. The NTT can be accessed at http://neurorehabilitation.m-iti.org/NTT/.

Our web services serve a threefold mission: instruction, training and feedback. The NTT site provides detailed instruction on how to use and operate the NTT. After giving informed consent, access to the NTT training is granted. The NTT runs as an embedded application on our site and delivers the training, logs data, and communicates with patients (Figure 1(D)). A remote data server is used to store log files after each training session containing relevant information such as the training date, user ID, data on the physical movements during training, game events, performance measures, and hardware configuration. Email services are used to communicate access codes and relevant user questionnaires after specific NTT training sessions.

2.1. NTT Game Training Scenario. The NTT web application was developed with the Python programming language using the open source game engine Panda3D (http://www .panda3d.org), which is maintained by Carnegie Mellon University. NTT is designed along neuroscientific and therapeutic guidelines for stroke rehabilitation based on a number of concepts: relevance of training to ADLs, neuroscientific principles of recovery, narrative, personalization or individualization, augmented feedback, and engagement.

2.1.1. Training Rational. After stroke, the recovery of the upper-limb functionality is essential for the recovery of the capacity of performing activities of daily living (ADL). 


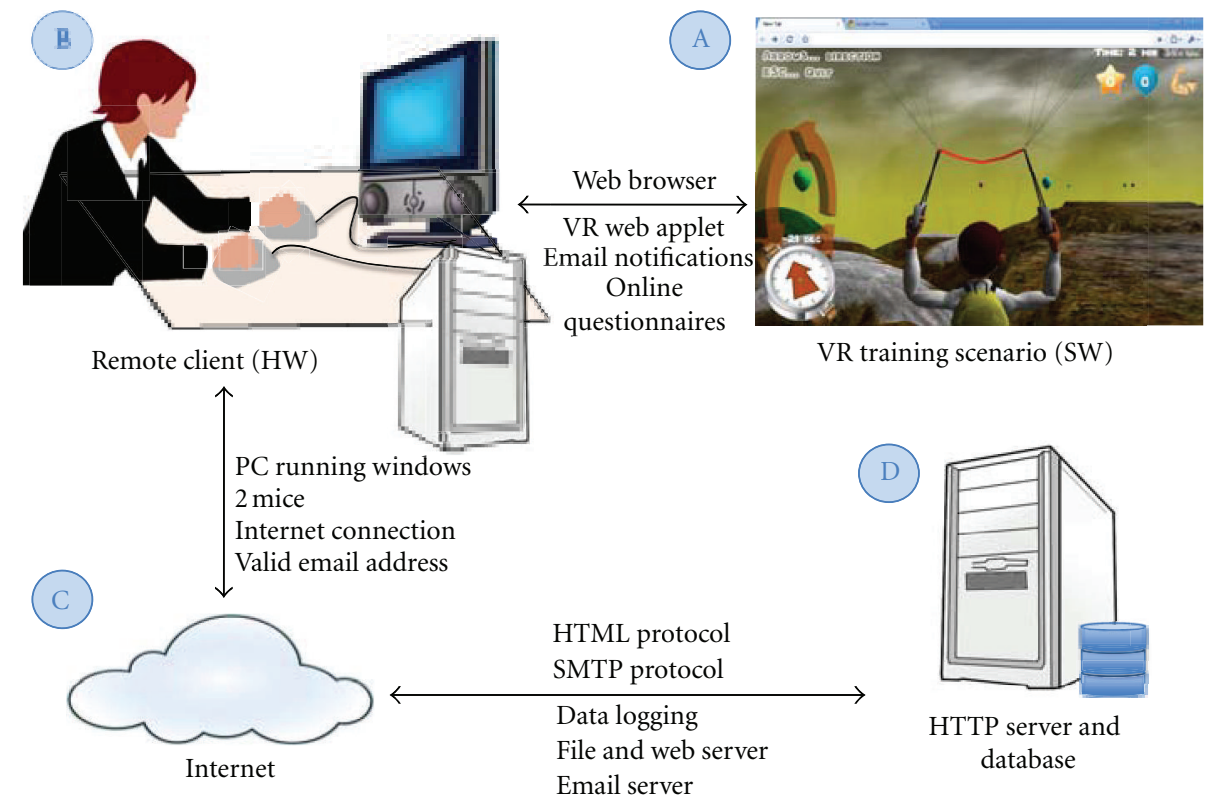

FIGURE 1: Neurorehabilitation Training Toolkit system architecture. (A) A web browser is used to access the application and its instruction at http://neurorehabilitation.m-iti.org/NTT/ (B) NTT can be executed on any modern PC equipped with two mice and an internet connection. (C) The NTT software is accessed freely from Internet, where the NTT servers are located. (D) A number of remote servers host the NTT site, the application itself, and are used to log user data.

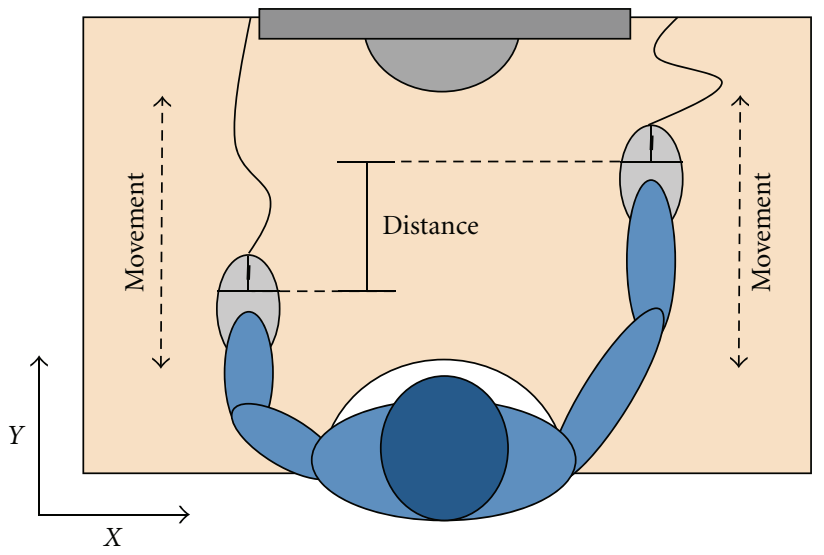

Figure 2: Sketch of the NTT-bilateral training task. Two mice track the position of right and left hands. The training consists of bilateral and coordinated displacement of the arms in the $Y$ axis to change the heading direction of the NTT-virtual glider. The distance between hands in the $Y$ axis defines the direction and the turning speed. See text for further information.

The ability of a stroke survivor to perform ADLs determines his/her level of independence to a great extent and therefore the need of specialized care, dependence on relatives, and societal burden [33]. Hence, the improvement of upperlimb function is a priority after stroke. Effective therapies for motor rehabilitation support the notion of training intensity, frequency, and task-specificity as being determinant outcome factors $[25,34,35]$. However, patients do not always have access to the ideal training frequency and intensity. In addition, the training specificity towards the needs of the patient appears to be of special relevance since it optimizes the training outcome [36]. Consequently, an effective upper-limb training paradigm should be based on training intensity, frequency, iteration, task specificity, and personalization of training to the patient's needs. NTT exploits the above-mentioned principles in a game-like experience where patients are confronted with a virtual scenario that requires them to repeatedly perform physical movements of varying intensity in order to complete a task. The NTT training task is performed on a tabletop (Figure 2).

With continuous research, more data about the poststroke brain mechanisms are becoming available which support the claim that neuronal plasticity is the main contributor for recovery [37-40]. Plasticity is a life-long property of the brain that allows cortical networks to reorganize and regain lost functionality, even many years after stroke $[11,41]$. Recent findings show how the reorganization of perilesional and contralesional cortical networks contributes to the recruitment of functional corticospinal fibers, which is vital for recovery $[39,42]$. Therefore, novel rehabilitation approaches should be designed to capitalize on brain plasticity to regain motor function by means of a functional reorganization of the cortical networks. It is widely established in the rehabilitation literature that training frequency and intensity are effective drivers for cortical reorganization [34, 35, 43]. However, more recent neuroscientific findings should also be integrated in current rehabilitation praxis. This is the case of approaches based on the Mirror Neuron System (MNS), a population of neurons located in premotor and parietal cortical areas that have particular properties that make them good candidates to mobilize plasticity, and for motor learning in general 
$[44,45]$. The MNS is also known as the action recognition system because of its properties. These neurons are active both during the performance of meaningful and goaloriented motor actions and the passive observation of those actions $[46,47]$. Thus, this is a convenient neural system that can be exploited to activate motor-related areas and therefore to mobilize cortical plasticity by means of the observation of motor actions [48, 49]. Several motor rehabilitation approaches capitalize on this system to enhance or speed up recovery $[32,50,51]$. In a previous randomized controlled trial, we have shown that a VR system based on this principle speeds up recovery [18] in terms of motor function as measured by the Fugl-Meyer scale [52] and in ADLs as assessed by the Chedoke Arm and Hand Activity Inventory (CAHAI) [53]. Consequently, and in line with our previous work, we have integrated those neuroscientific aspects in the NTT by means of the presentation of a virtual character whose limbs perform meaningful goal-oriented actions that are triggered by and correlated with the physical movements of the arms of the patient. The combination of VR and goaloriented actions has already been shown to activate the MNS and related motor areas, therefore facilitating functional cortical reorganization [50].

2.1.2. Training Task. In its current form, the NTT training task requires patients to be able to read, and not to have major cognitive deficits and seizures, sensory aphasias, or other perceptual problems that could impede the understanding of the task. Our training consists of a bilateral task that requires practicing range of motion and movement coordination. Several reasons support the choice of this particular task. First, a home-based training has to allow for a gradual functional integration and use of the paretic arm in the completion of tasks. Thus, our bilateral training task allows the patient to support the paretic arm with the nonparetic one when the task is too demanding. As such, our training task is also appropriate for hemiplegic patients with severely reduced mobility or motor control. Second, although evidence on the advantage of bilateral training when compared to unilateral training is limited [54], there are a number of findings that support its use [55, 56]. For instance, nowadays it is widely accepted that bimanual coordination is a largely distributed brain process, and therefore its training engages motor areas to a larger extent [57]. Moreover, since most patients manifest some degree of bimanual coordination deficit, bimanual coordination training is recommended to be part of any rehabilitation program [58]. Further, there is increasing evidence that bilateral training has a particularly beneficial effect in patients with low Fugl-Meyer motor scores, whereas it is as effective as unilateral training in well-recovered patients $[55,59]$.

The NTT bimanual training exercise is performed on a tabletop, providing arm support against gravity, what widens the spectrum of patients who can use it. The patient's hand movements are tracked by means of two computer mice that the patient is manipulating (Figure 2). The interaction with real objects has been shown to elicit better kinematics [60]. The physical movements of the patient are then used to control the movements of the arms of an avatar that is displayed on the computer's screen. The avatar arms control the steering direction of a glider that flies forward at a constant speed, accumulating collectable objects. The turning speed of the glider $(\alpha \mathrm{deg} / \mathrm{sec})$ is defined as a function of the distance between mice (Figure 2):

$$
\alpha=\text { gain } \cdot\left(Y_{\text {Right }}-Y_{\text {Left }}\right),
$$

where $Y_{\text {Right }}$ and $Y_{\text {Left }}$ indicate the $Y$ position of right and left mice, respectively, and gain is a factor that modulates the turning speed of the avatar.

2.1.3. Gaming Concepts. The glider control task was chosen due to the bimanual and intuitive nature of the control mechanism, the built-in control system that allows supporting the paretic arm actions with the nonparetic arm, the slow movement dynamics, and the pleasantness of a flying experience.

Improving on previous work, we decided to add a narrative element to the NTT since a flat and static virtual training task can make training monotonous and eventually limit the patient's engagement. Consequently, NTT exploits a simple narrative structure to build a story around the training task to increase the engagement of patients, facilitate the comprehension of the training objectives, and, most importantly, to deliver a clear sense of progress. This simple narrative is based on Freytag's classic concept of play and counter-play [61]. Play in our case describes how our player affects the world (an exciting force or rise), and counter-play how the world affects the player (a tragic force or decay). We created a sequence of plays and counter-plays that narrate the journey of our virtual character through an unknown environment that culminates with him finding home. The game structure includes a tutorial of the game, and a series of game levels/environments that lead the way to a narrative climax when home is reached (Figure 3(a)). In order to reach home, the avatar has to collect flying objects that enable him to complete the different game levels. Different game elements are used to shape the narrative curve; these include the task difficulty, presence of control disturbances, the choice of the color palette, the design of the environment itself, the choice of music and its rhythm, and the usage of the view/camera perspective.

In addition to the narrative component, the NTT makes extensive use of feedback using multiple and redundant feedback modalities to ensure that the patient understands the task and is positively rewarded for his/her accomplishments $[28,62]$. For instance, on-screen pictorial instructions and a compass are used to indicate the direction and physical movements that are needed in order to get to the target. The current item to be collected is clearly indicated by a large moving arrow. To help the patient in the task, both the target arrow and the compass head turn from red into green when the avatar is correctly aligned in direction to the target. Every collected item is rewarded with a positive visual and auditory feedback. Collectable items are divided into easy (balloons) and hard (stars) ones, which are counted as separate scores. Finally, the total distance moved by the arms during training 


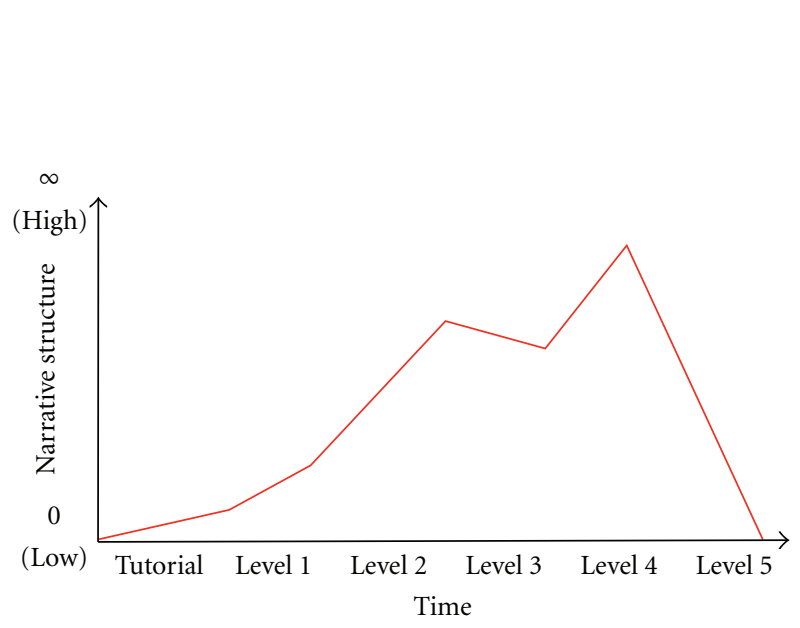

(a)

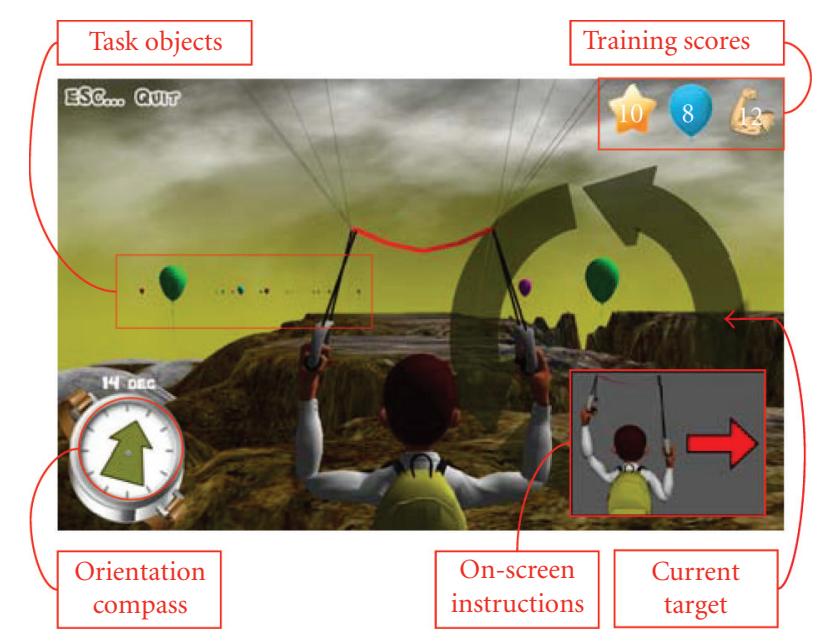

(b)

FIGURE 3: Game design elements of the Neurorehabilitation Training Toolkit (NTT). (a) Curve describing the narrative structure of the NTT where the different game levels are assigned to a narrative (interest) value. (b) Snapshot of the NTT training scenario during a game play. Task and feedback elements are indicated.

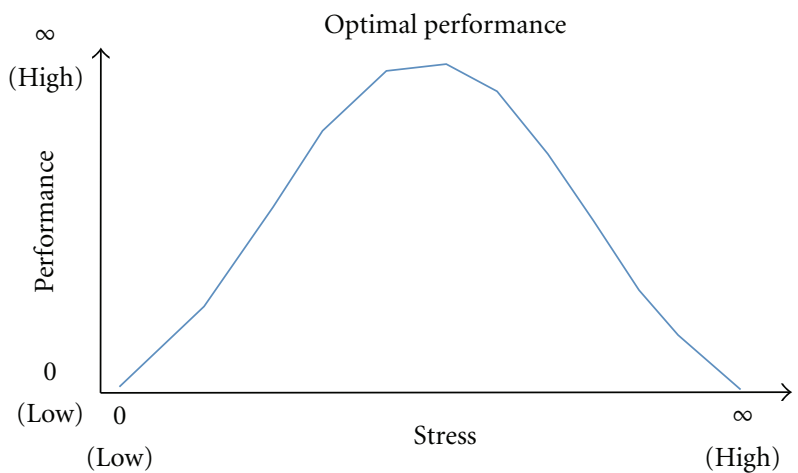

(a)

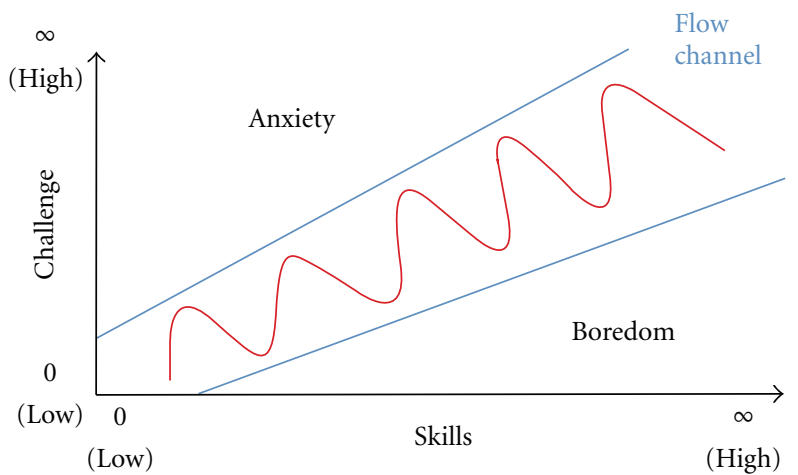

(b)

FIgURE 4: Design principles of the personalization of training with the NTT. (a) Yerkes-Dodson law established an inverted U-curve dependence between stress level and task performance. (b) Flow theory places the maximum user engagement in the balance between skills and challenge in a given task, described as the flow channel. See text for further details.

is continuously computed and updated on a screen counter (Figure 3(b)).

2.1.4. Personalization of Training. Since 1908, when Yerkes and Dodson discovered the relation between induced stress and task-learning performance in mice, it became clear that optimal learning is only achieved at intermediate stress levels [63]. Low and high levels of stress result in low performance levels (Figure 4(a)). These results were later replicated with humans in different research domains [64, 65]. Therefore, training systems such as the NTT that aim at optimizing motor learning and performance would benefit from incorporating this concept. Furthermore, the YerkesDodson law is consistent with Csikszentmihalyi's flow theory that describes flow as "a state of peak enjoyment, energetic focus, and creative concentration by people engaged in adult play" [66]. Flow theory describes user experience during play (anxiety, boredom, and flow) depending on the challenge experienced and skill required. Flow is placed at the right balance between user skills and level of challenge (Figure 4(b)). Therefore, the NTT requires a built-in capacity to adjust the training parameters to an intermediate difficulty level, to avoid stressful/boring configurations, maximize learning, and to maximize user engagement as described by flow theory.

Our training parameters can be easily modified given that the NTT training task is implemented as a computerbased VR training task. A total of 4 task-related parameters (independent variables) define the training specifics: speed (the forward flying speed of the avatar), turning (turning speed of the avatar described as gain in (1)), acceptance radius (how close one has to be to an object to collect it), and distance (distance between collectable items). Therefore, the overall objective of the personalization of these parameters is to allow the NTT to provide its users with a transparent, 
automated, autonomous, and unsupervised personalization of training. Consequently, this personalization of training allows the NTT to support both optimized training (Yerkes Dodson law) and better user engagement (Flow theory). Additionally, the training parameters can also serve the purpose of monitoring motor improvements over time. The main research objectives of this work are to analyze the interactions between game parameters, relate them to quantifiable training objectives such as range of motion, coordination or movement smoothness, and to propose a model to automate the parameter selection for optimal training.

2.1.5. Deployment and Built-In Protocol. The most novel and possibly the strongest aspect of a rehabilitation tool such as the NTT is that it is accessible for millions of patients, anywhere in the world. This poses many opportunities but also some challenges. The most important challenge is probably the lack of a direct control on the usage of the NTT. This means that aspects such as instruction, training duration and parameters, data collection (questionnaires and game data), and informed consents have to be dealt with directly and automatically from the NTT site and the application itself. Thus, the NTT web site guides the user through a simple step-by-step tutorial on how to execute the NTT applet. Additionally, a video tutorial has been created to explain the usage of the Neurorehabilitation Training Toolkit. Only after giving informed consent for participation in a research study, the NTT application itself is launched.

The use of NTT requires a working email address to receive an access password and questionnaires. Consistent with previous studies, the duration of the training is set to 20 minutes with a suggested training frequency of three times per week [18]. The patients' behavioral data are automatically collected by the NTT.

Two self-report questionnaires were designed to gather demographic data, assess user experience and self-assessed improvements. The questionnaire on user experience consists of 13 questions that are presented in the format of a 5 -point Likert scale where patients report their level of agreement/disagreement with respect to a number of statements. Self-assessed improvements in ADLs are evaluated by means of the modified Barthel Index questionnaire [67]. Questionnaires are sent via email in session 1 (start of training), 6 (2 weeks), 12 (one month), and so on. Questionnaires are displayed as web pages, and user data is collected using Google forms technology (Google Inc., Mountain View, CA, USA).

\subsection{Pilot Study}

2.2.1. Purpose and Procedures. In order to study the NTT properties and to develop a model for the automated update of the game parameters, a number of experiments were performed. 10 right-handed healthy naive participants aged 23 to 45 years $(\mathrm{M}=30.5, \mathrm{SD}=6.69)$ from the University of Madeira were recruited to use the NTT. All participants were using the NTT for their first time, and the experiments were performed in a laboratory setting that always used the same
TABLE 1: Exploratory parameter space used in the NTT pilot-testing phase.

\begin{tabular}{lcc}
\hline & Minimum & Maximum \\
\hline Speed (flying speed) & $4 \mathrm{~m} / \mathrm{s}$ & $80 \mathrm{~m} / \mathrm{s}$ \\
Turning (turning speed) & $15 \mathrm{deg} / \mathrm{s}$ & $80 \mathrm{deg} / \mathrm{s}$ \\
Acceptance radius & $1 \mathrm{~m}$ & $10 \mathrm{~m}$ \\
Distance (between targets) & $10 \mathrm{~m}$ & $40 \mathrm{~m}$ \\
\hline
\end{tabular}

hardware configuration. This pilot study followed standard approved guidelines and conformed to current EU and Portuguese legislation. All users gave their informed consent to participate in this study. Two identical mice (ems069i00, eblue, Hong Kong) were used for tracking purposes. A total of 120 minutes of data were collected where participants played several "training levels" with random values for the different game parameters described in Section 2.1.4 (speed, turning, acceptance radius, and distance). These parameters were selected within the exploratory space described in Table 1, which was defined to range from an easy and controllable task to a difficult and highly demanding psychomotor task.

Complete log files of all game events, parameters, and all motor actions performed by participants were collected. These log files were stored in text format and subsequently imported into Matlab 2008a (MathWorks Inc., Natick, MA, USA) to perform the data analyses.

2.2.2. Accuracy of the Capturing Devices. Computer mice are inertial sensors, devices that are only capable of measuring instantaneous changes in position as opposed to absolute position. As a consequence, their position is calculated as the accumulated instantaneous changes in position, what makes them subject to drift. In order to assess the extent and impact of their drift, we have quantified it using AnTS, a camerabased vision tracking system that provides an absolute coordinate system free of drift (http://neurorehabilitation .m-iti.org/software/) $[32,68]$. The AnTS tracking system was set up to track the position of the mouse on the table top while performing a NTT training session. The data from AnTS, captured using a camera with $640 \times 480$ pixel resolution and with a position accuracy of about $1 \mathrm{~mm}$, was recorded synchronously with the reading from the mice. After the NTT session, we quantified the percentage of drift by comparing the position reconstructed with the mouse data and that coming from AnTS, it corresponded to $0.56 \%$ of the total distance travelled.

2.2.3. Kinematic Analysis of Upper-Limb Function. Relevant kinematic parameters for post-stroke rehabilitation include smoothness of movement, range of motion, and total arm displacement [60]. There is evidence showing that nonparetic arm kinematics and kinetics of patients do not significantly differ from those of normal subjects [69]. Thus, bilateral measurements from paretic and nonparetic arms become extremely valuable to assess and monitor improvement over time. Further, the NTT training task is unique because it allows us to assess characteristics such as arm 
coordination and contribution of each arm. Thus, data gathered by the NTT could allow for a kinematic analysis of upper-limb function, providing comparative data on each patient's paretic versus nonparetic arm. We performed a first pilot evaluation with healthy participants to assess the adequacy and validity of the NTT as a tool to assess the above-mentioned kinematic measures in healthy users.

Smoothness Index. Consistent with previous research, we define a Smoothness Index (SI) that quantifies the regularity and smoothness of a particular movement trajectory [69]. This measure allows us to assess the level of fine movement control of the NTT users for each arm independently. The smoothness of a movement depends on the number of segments in which it can be divided. Here we consider movement segments as those segments in between movement accelerations and decelerations. Therefore, the SI is computed from an analysis of the speed profile of a movement. We define SI as the count of the number of local minima in the speed profile for a specific action. Thus, lower SI values indicate movements with a smoother velocity profile, whereas higher values indicate those movements with more accelerations changes. Before the calculation of the SI, mice data are prefiltered with a Gaussian window filter of $1 \mathrm{sec}$ length to reduce the amount of noise.

Arm Displacement. In order to display feedback about the actual work that has been realized, NTT also computes the accumulated distance that each hand has been displaced during training (Figure 3, training scores). This is a measure of actual physical exercise and allows us to assess the exact amount of physical movement that was needed by each user to complete the training tasks.

Range of Motion. Another relevant kinematic measure, related to the actual amount of physical movement, is Range of Motion (ROM). There is evidence that suggests that ROM can be used as a predictor of functional outcome in ADL [70]. As such, being able to automatically assess and monitor ROM throughout the training process can become a rather valuable feature of the NTT, particularly when complemented with the Barthel Index assessment for performance in ADLs [67]. The ROM that is measured by the NTT results from the combined action of shoulder and elbow (extension and flexion) on the end effector, the hand, which is tracked with the mouse. ROM captures aspects of the movement dynamics, providing information on functional arm aperture that other measures cannot provide. In the case of stroke patients, a limited ROM does not necessarily indicate limited movement within the working ROM. Our operational measure of functional ROM-the ROM being effectively used during the NTT training-is computed in $\mathrm{cm}$ from consecutive arm extensions and flexions as measured by mice.

Arm Coordination and Arm Contribution. As opposed to other rehabilitation approaches, NTT allows for an automated and objective quantification of functional outcomes, some of which are not possible to quantify in real-world tasks without the aid of interactive technologies. One particular example of this unique capability of the NTT is the ability to quantify arm coordination. During NTT training, and because of the bilateral nature of its training, physical movements are expected to be symmetrical movements performed simultaneously with both arms. We therefore quantify arm coordination as the absolute value of the correlation coefficient between the data from both mice. Consequently, the computation of the correlation coefficient of perfectly coordinated movements would result in a coefficient equal to 1 . Our quantification of movement coordination is particularly advantageous for our purpose since the correlation operation is insensitive to the actual gain of the movement. Namely, perfectly correlated (coordinated) movements are not required to be of the same amount but should follow the same temporal profile. This means that the NTT enables us to separately assess movement coordination and arm contribution to the training task. The later-separate arm contribution-is computed by assessing the particular involvement of each arm in the steering of the avatar. Since the avatar's heading direction is driven by the differential in position of the two mice, the training can be performed with dissimilar involvement of the paretic and nonparetic arms (Figure 2). Thus, the contribution of each arm is computed as the correlation coefficient between the arm movement trajectories over time and the effective heading direction changes as described by (1). So, a low correlation coefficient will be obtained for an arm with reduced contribution to the bilateral control task.

Modeling. If successful, the ability of the NTT to provide us with relevant behavioral information about the patient's motor capabilities will allow us to assess the impact of the NTT on different aspects of motor recovery. Here we introduce how the NTT can be tailored to automatically selfadapt depending on the user's needs. In order to do so, we first have to quantify the relationship between our kinematic analysis of upper-limb function and the NTT training parameters. For that reason we use a multiple regression modeling approach. Consistent with previous work on psychometrics, our model considers all game parameters, its second-order terms, and first-order interactions [32]. In our case, we propose to model each of the previously described kinematic measures (Section 2.2.3) in the following way:

$$
\begin{aligned}
\text { Kinematic measure }= & c_{0}+c_{1} * \text { speed }+c_{2} * \text { turning } \\
& +c_{3} * \text { acceptance }+c_{4} * \text { distance } \\
& +c_{5} * \text { speed } * \text { turning } \\
& +c_{6} * \text { speed } * \text { acceptance } \\
& +c_{7} * \text { speed } * \text { distance } \\
& +c_{8} * \text { turning } * \text { acceptance } \\
& +c_{9} * \text { turning } * \text { distance }
\end{aligned}
$$




$$
\begin{aligned}
& +c_{10} * \text { distance } * \text { acceptance } \\
& +c_{11} * \text { speed }^{2}+c_{12} * \text { turning }^{2} \\
& +c_{13} * \text { acceptance }^{2}+c_{14} * \text { distance }^{2}
\end{aligned}
$$

where kinematic measure is the dependent variable (either movement smoothness, range of motion, arm displacement, or arm coordination); speed, turning, acceptance, and distance are the independent variables set by the NTT during our pilot-testing experiment, and the $c_{i}$ constants are the coefficients resulting from the multiple regression analysis.

Besides the kinematic modeling approach, a parallel system is established to maximize challenge and engagement and therefore potentiate the highest performance level and flow. This is achieved by means of a time race $[62,71]$ during which NTT monitors the efficiency of the patient's actions by assessing his/her performance at every task completion (item collection) (Figure 5, efficiency assessment). Given that the flight speed and item distance are known training parameters to the NTT, it is possible to define efficiency in the following way:

$$
\text { Efficiency }=\frac{\text { actual task completion time }}{\text { ideal task completion time }} \text {. }
$$

Efficiency is computed at every task completion/object collection, which enables estimating how much time is needed by the patient on each task. Thus, the time race is implemented by providing every object with a timer that indicates how much time is left to collect it. The timer is based on the patient's efficiency data from previously collected items, being modulated by a Gaussian noise function $(\mathrm{M}=1, \mathrm{SD}=$ 0.2) (Figure 5, task time). Targets that allow more than the average time needed by the patient are presented as balloons (easy), and targets requiring less time are presented as stars (difficult). If the task is not completed within the time window, the target disappears and the timer is reset to a longer time as determined by the patient's mean efficiency (3). On one hand, this time race strategy allows personalizing the challenge of the task to the particular skills of the patient [62]. This design allows us to change task difficulty without interfering with the training parameters, which in our case determine the training objectives. On the other hand, training parameters (speed, turning, acceptance, and distance) are only updated when the patient's efficiency increases or decreases (3). Hence, the more efficient the patient is on the task, the higher the demands in terms of training objectives. Consequently, our personalization algorithm consists of two parallel loops that shape challenge and training parameters to provide optimized and enjoyable training.

\section{Results}

As described above, the NTT was evaluated with 10 righthanded healthy naive participants. As expected, healthy participants present a low Smoothness Index SI $(M D=5)$, with

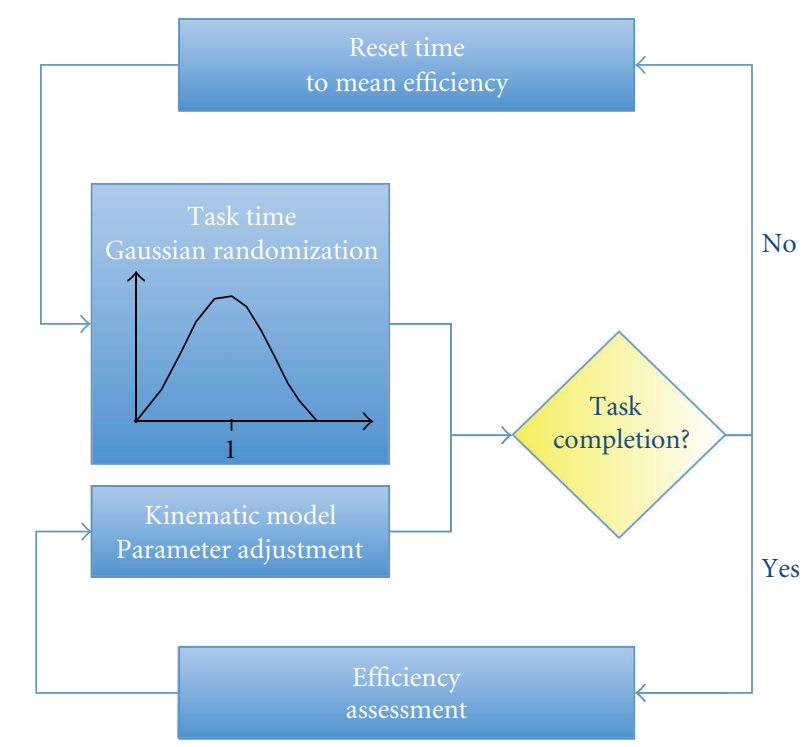

FIGURE 5: NTT personalization process. The adaptation of the training parameters is realized according to the patient's efficiency in the task. A kinematic model maps training objectives to individual NTT parameters. In parallel, a timer is used to create a time-race task. See text for further information.

no significant difference between dominant and nondominant arms (Wilcoxon signed-ranks test, $Z=-.0212, P=$ .9831) (Figure 6). A similar SI interquartile range (IQR) for dominant and nondominant arms is found (6.5 and 6, resp.).

Example data on arm displacement from participant number 1 is shown in Figure 7. A group analysis shows a median hand displacement of $23.25 \mathrm{~cm}$ per item collected, moving the dominant arm significantly less than the nondominant arm (Wilcoxon Signed-ranks test, $Z=$ 13.5642, $P<.0001)$.

Consistent with the arm displacement data, a paired sample $t$-test analysis revealed that the dominant arm uses a lower functional $\operatorname{ROM}(\mathrm{M}=23.38, \mathrm{SD}=16.14 \mathrm{~cm})$ than the nondominant arm for healthy participants $(\mathrm{M}=27.18$, $\mathrm{SD}=18.12 \mathrm{~cm}), t(403)=-4.6924, P<.0001$ (Figure 8$).$

The investigation of arm coordination in healthy participants reveals a high degree of coordination, that is, high correlation values between the movement trajectories of the arms during the training task $(\mathrm{M}=.8143, \mathrm{SD}=.2433)$. This is consistent with the high degree of motor control of this population of users (Figure 9). Interestingly, the arm contribution analysis of the NTT training task- which assesses the impact of the movement of each arm in the steering of the avatar-reveals larger contributions $(t(9)=$ $-8.5925, P<.0001)$ for dominant arms $(\mathrm{M}=.5631, \mathrm{SD}=$ $.1872)$ than for nondominant arms $(\mathrm{M}=.5095, \mathrm{SD}=$ .2333) (see Section 2.2.3 for the details on the quantification of arm coordination and contribution). While this result is consistent with what is expected from dominant arms, in previous measures, we observed lower displacement and ROM for the dominant arm. This indicates that the dominant arm does not only contribute more, but it is also 


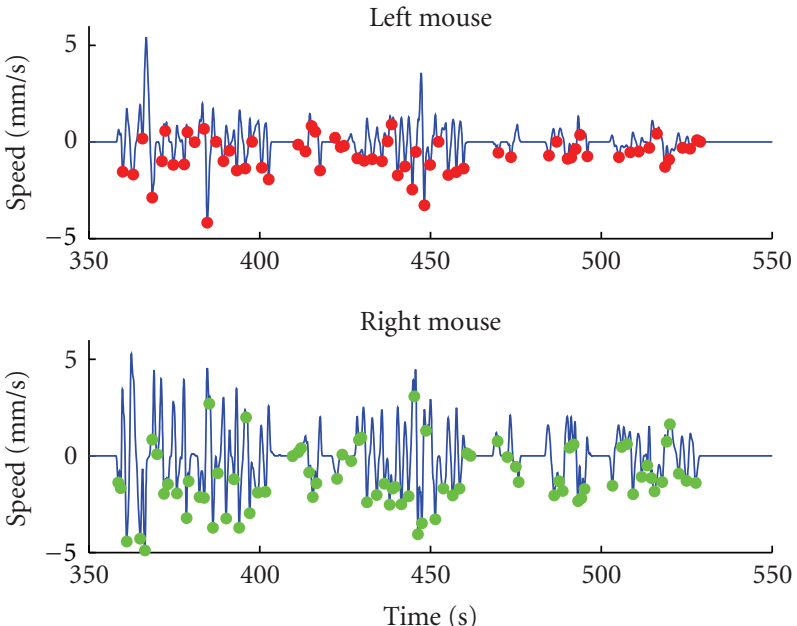

(a)

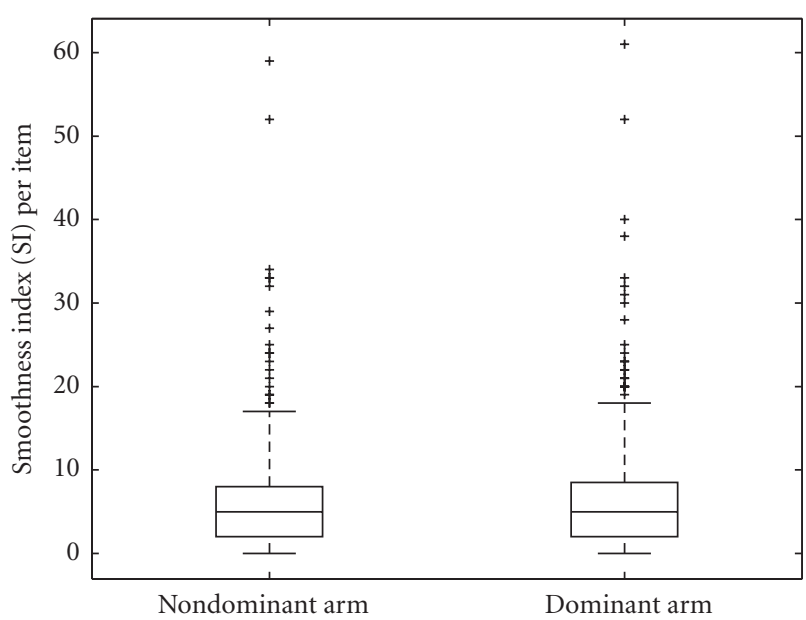

(b)

Figure 6: Analysis of movement smoothness by means of the Smoothness Index (SI). (a) Sample speed profiles of the movements tracked by both NTT mice. The number of movement segments contained in a specific movement trajectory is computed by locating the speed local minima (red and green dots). (b) SI is computed for each arm and for each item collected in the virtual training environment. Boxplot from 10 healthy participants. For more information see text.

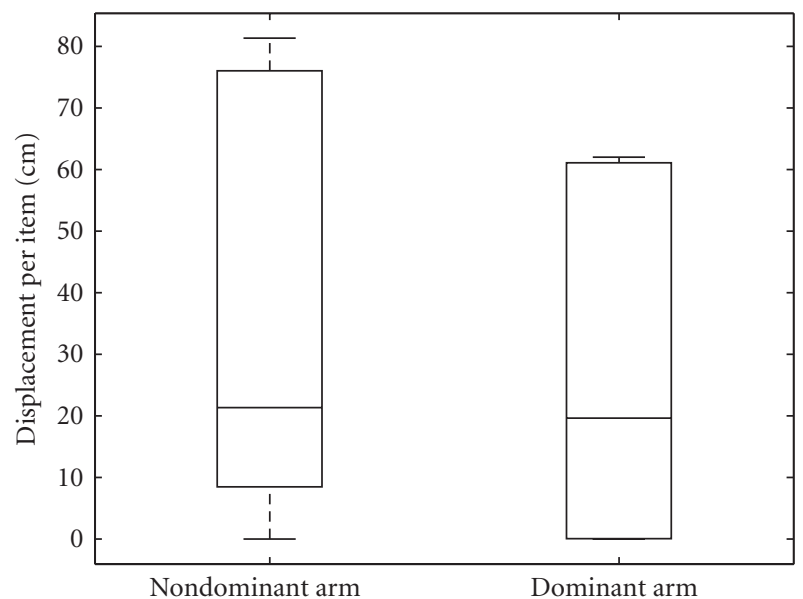

Figure 7: Arm displacement on the tabletop $(\mathrm{cm})$ per item collected during a NTT training session. Data from healthy participant number 1 .

more precise in its actions, requiring less movement and ROM to accomplish the task.

Figure 9 shows that the NTT is capable of assessing different aspects of the movement kinematics when used by healthy users. Consequently, if we can assess how the NTT training parameters relate to the actual resulting physical exercise and movement kinematics on the tabletop, we can optimize the NTT training parameters accordingly to deliver appropriate and personalized training. Subsequently, we used these quantitative data on movement kinematics combined with modeling techniques to establish statistical relationships between the training parameters used and the actual physical exercise associated with them. We performed four separate multiple regression analyses to find out how

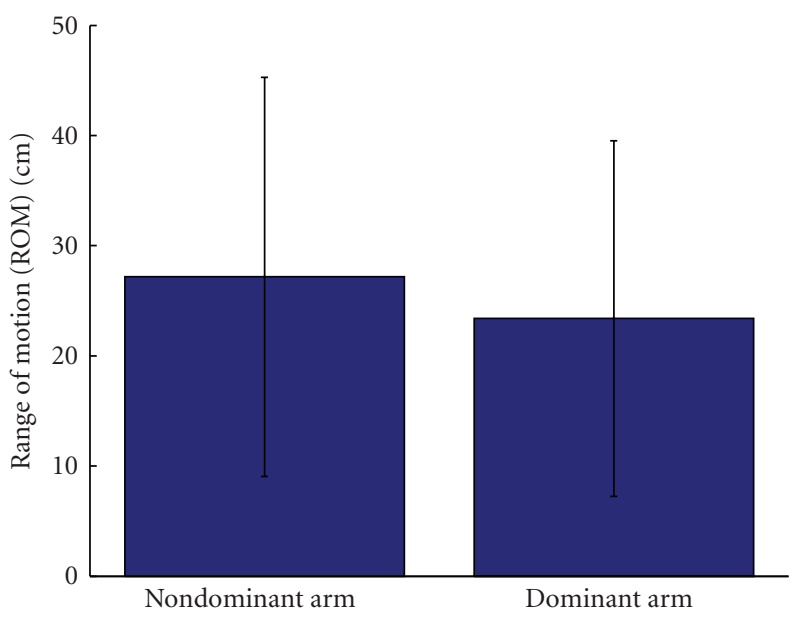

Figure 8: Range of Motion (ROM) usage during NTT training. Data consist of 404 data samples from the 10 healthy participants. Bar indicates mean ROM and error bars SD.

the NTT training parameters contribute to the changes observed in the previously determined kinematic measures. Using the data from our healthy participants, we have identified the training parameters, the first-order interactions, and the second-order parameters that contribute to the movement smoothness, range of motion, arm displacement, and arm coordination (Table 2). The exact model coefficients described in (2) and related statistics are shown in Table 3 (see supplementary material available online at doi:10.1155/2012/802157).

From the multiple regression analysis we can conclude that not all training parameters contribute to all movement kinematics. This means that, depending on our training 
TABLE 2: Table of significances of the multiple regression analyses. Crosses indicate terms that have a significant contribution to the kinematic measure and thus are taken into account in our modeling purposes. $s, t, a$, and $d$ refer to the training variables flying speed, turning speed, acceptance radius, and distance, respectively. See Table 3 for further details on the model coefficients and related statistics.

\begin{tabular}{|c|c|c|c|c|c|c|c|c|c|c|c|c|c|c|}
\hline & $s$ & $t$ & $a$ & $d$ & $s * t$ & $s * a$ & $s * d$ & $t * a$ & $t * d$ & $a * d$ & $s^{2}$ & $t^{2}$ & $a^{2}$ & $d^{2}$ \\
\hline Movement smoothness & & & & $x$ & & & & & & & & & & $\times$ \\
\hline Range of motion & & $x$ & & & & $x$ & & & & & $x$ & $\times$ & $x$ & \\
\hline Arm displacement & & & & & & & & & & $\times$ & & & & \\
\hline Arm coordination & & & & & $x$ & $x$ & & & & & & & & $\times$ \\
\hline
\end{tabular}

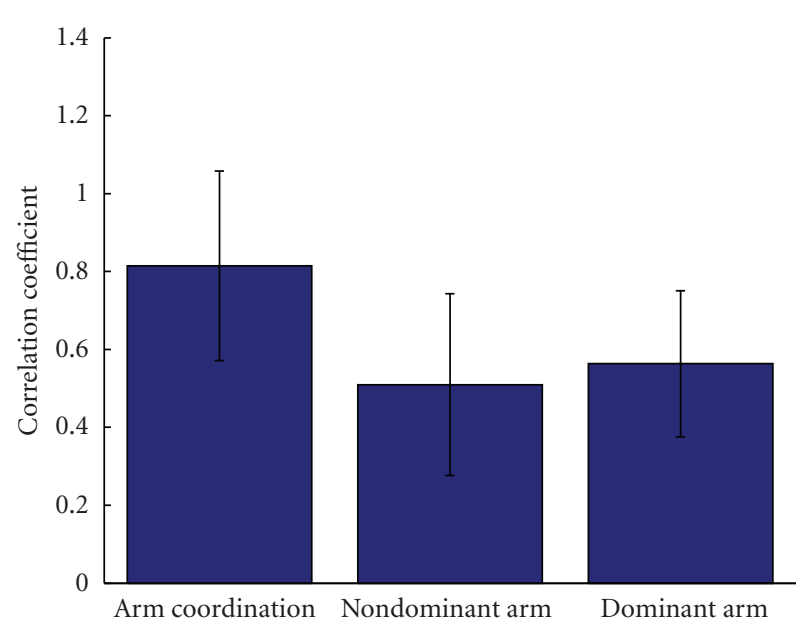

FIGURE 9: Arm coordination and contribution analysis for the NTT training task. Data from 10 healthy participants. Bars indicate the mean and error bars the SD.

objectives, a different set of parameters need to be changed to affect the user's movement kinematics. Thus, according to the relations established in Table 2, we have implemented a personalization training algorithm that can adjust the training parameters depending on the training objectives set by the user (Figure 5). In this algorithm for the adaptation of training, only a subset of the training parameters will be adjusted during training depending on whether the user needs to train movement smoothness, ROM, arm displacement, arm coordination, or any combination of these. The selection of the training objectives will therefore determine the kinematic models to be used during an NTT session (Figure 5, kinematic model).

\section{Conclusions and Discussion}

In this paper we presented the Neurorehabilitation Training Toolkit (NTT), a low-cost neurorehabilitation system that aims at offering an alternative for continued at-home rehabilitation after stroke. The NTT is designed along neuroscientific guidelines as well as game design principles to drive motor recovery, while maximizing engagement and motivational factors. Additionally, it is designed to be compatible with any modern PC that runs Windows O.S. and has an Internet connection. Because of its architecture and design, the NTT is a unique rehabilitation system. By capitalizing on broadly available home technology, the NTT is a free rehabilitation tool that is accessible worldwide via a web browser. This feature makes it immediately available for large-scale deployment, having an enormous potential as a motor training solution for patients in remote areas, with low income, low mobility, or without access to rehabilitation facilities. Furthermore, it is designed to serve as an athome complement to other training or simply for continued rehabilitation after hospital discharge. Since NTT is accessed via a web browser, it does not have to be installed on the patient's computer and it is easily upgradeable, allowing patients to increase their portfolio of training games. NTT is a completely self-contained training system that does not require the intervention of a therapist or specialist. Further, NTT is a safe system completely based on noninvasive technologies, making it a suitable solution for unsupervised and at-home use. Based on the psychometric analysis presented here, the NTT is designed to automatically adjust its training parameters to the training. More concretely, NTT allows training movement smoothness, range of motion, arm displacement, arm coordination, or any combination of these based on the presented kinematic models.

As opposed to other approaches using technologies such as robotic devices, custom-made tracking technologies, brain-computer interfaces, data gloves, or expensive commercial hardware $[16,32,51,72-75]$, the NTT only requires a PC, a pair of computer mice and an Internet connection. This advantage of the NTT, which lowers the accessibility threshold for at-home rehabilitation training technologies, also represents one of its most important challenges. For instance, the lack of a supervised setting and the absence of complete upper-body tracking represent a limitation that more sophisticated systems do not suffer [16, 18, 75-77]. More specifically, the NTT kinematic analyses are based on the end effector. However, despite this caveat, the NTT is able to contribute with a detailed and functional analysis of training task performance. As reported in this paper, outcomes such as ROM, arm displacement, arm coordination, and individual arm contribution to the task objectives are unique assessment properties of the NTT. Our study with healthy participants has shown a precise quantification of ROM, coordination, arm contribution, and smoothness of movement, while also being able to detect handedness. Interestingly, the data revealed differences in ROM and arm contribution as result of handedness but no differences in movement smoothness. Compared to the nondominant arm, the dominant arm displayed a significant reduction of ROM while simultaneously showing an increased contribution in 
task performance. In the particular case of patients, ROM and accumulated displacement are expected to be more informative since they reveal different aspects of movement kinematics (arm aperture and amount of movement), which are not necessarily dependent. Although we have shown a high accuracy assessing functional outcome in healthy users, more research is needed to understand the limitations of this system when used by elderly and stroke patients. Given the nature of the bilateral training task and the possibility of supporting the actions of the paretic arm with the nonparetic arm, the use of compensatory movements is expected to be reduced. Nevertheless, we need to understand the extent of the use of compensatory movements and its effect in the assessed movement kinematics, since combined trunk, shoulder and elbow movements can contribute to effective hand movements. Similarly, the presented kinematic models generated with healthy user data need to be further validated in a trial with patients.

Despite the limitations of the presented psychometric modeling, the NTT goes beyond the adaptive and personalized training of previous work, allowing for a novel individualized and parametric training of several functional outcomes [18]. Finally, the automatic data-collection capability of the NTT and its questionnaire system enable a large-scale deployment to quantify its impact. We believe that rehabilitation technologies will continue to move in this direction, from custom and more expensive hardware-based solutions to low-cost systems that can be used at the patient's home $[15,16,31]$. The presented study is the first step that validates the design and technological aspects of the NTT with healthy users. In the next phase, it will be deployed and validated with stroke patients at their homes. The NTT, with its novel design principles and assessment capabilities, represents another step towards consolidation and at-home deployment of these technologies.

\section{Acknowledgments}

Support for this research was provided by the Fundação para a Ciência e Tecnologia (Portuguese Foundation for Science and Technology) through the Carnegie Mellon Portugal Program under Grant CMU-Pt/0004/2007.

\section{References}

[1] K. Strong, C. Mathers, and R. Bonita, "Preventing stroke: saving lives around the world," The Lancet Neurology, vol. 6, no. 2, pp. 182-187, 2007.

[2] World Health Organization, "The global burden of disease: 2004 update," Tech. Rep., World Health Organization, Geneva, Switzerland, 2008.

[3] A. di Carlo, "Human and economic burden of stroke," Age and Ageing, vol. 38, no. 1, pp. 4-5, 2009.

[4] L. Ayerbe, S. Ayis, A. G. Rudd, P. U. Heuschmann, and C. D. A. Wolfe, "Natural history, predictors, and associations of depression 5 years after stroke: the South London Stroke Register," Stroke, vol. 42, no. 7, pp. 1907-1911, 2011.

[5] S. A. Thomas and N. B. Lincoln, "Factors relating to depression after stroke," British Journal of Clinical Psychology, vol. 45, no. 1, pp. 49-61, 2006.
[6] C. D. Mathers and D. Loncar, "Projections of global mortality and burden of disease from 2002 to 2030," PLoS Medicine, vol. 3, no. 11, p. e442, 2006.

[7] A. S. Kim and S. C. Johnston, "Global variation in the relative burden of stroke and ischemic heart disease," Circulation, vol. 124, no. 3, pp. 314-323, 2011.

[8] P. Langhorne, L. Widen-Holmqvist, G. Taylor et al., "Early supported discharge after stroke," Journal of Rehabilitation Medicine, vol. 39, no. 2, pp. 103-108, 2007.

[9] T. Larsen, T. S. Olsen, and J. Sorensen, "Early home-supported discharge of stroke patients: a health technology assessment," International Journal of Technology Assessment in Health Care, vol. 22, no. 3, pp. 313-320, 2006.

[10] H. S. Jorgensen, H. Nakayama, H. O. Raaschou, J. Vive-Larsen, M. Stoier, and T. S. Olsen, "Outcome and time course of recovery in stroke. Part II: time course of recovery. The Copenhagen Stroke study," Archives of Physical Medicine and Rehabilitation, vol. 76, no. 5, pp. 406-412, 1995.

[11] T. Hodics, L. G. Cohen, and S. C. Cramer, "Functional imaging of intervention effects in stroke motor rehabilitation," Archives of Physical Medicine and Rehabilitation, vol. 87, no. 12, supplement 2, pp. 36-42, 2006.

[12] S. J. Page, D. R. Gater, and P. Bach-Y-Rita, "Reconsidering the motor recovery plateau in stroke rehabilitation," Archives of Physical Medicine and Rehabilitation, vol. 85, no. 8, pp. 13771381, 2004.

[13] I. G. L. van de Port, G. Kwakkel, I. van Wijk, and E. Lindeman, "Susceptibility to deterioration of mobility long-term after stroke: a prospective cohort study," Stroke, vol. 37, no. 1, pp. 167-171, 2006.

[14] L. Piron, A. Turolla, M. Agostini et al., "Exercises for paretic upper limb after stroke: a combined virtual-reality and telemedicine approach," Journal of Rehabilitation Medicine, vol. 41, no. 12, pp. 1016-1020, 2009.

[15] M. Sivak, C. Mavroidis, and M. K. Holden, "Design of a low cost multiple user virtual environment for rehabilitation (MUVER) of patients with stroke," Studies in Health Technology and Informatics, vol. 142, pp. 319-324, 2009.

[16] M. J. Johnson, Y. Shakya, E. Strachota, and S. I. Ahamed, "Low-cost monitoring of patients during unsupervised robot/computer assisted motivating stroke rehabilitation," Biomedizinische Technik, vol. 56, no. 1, pp. 5-9, 2011.

[17] E. B. Brokaw, I. Black, R. J. Holley, and P. S. Lum, "Hand Spring Operated Movement Enhancer (HandSOME): a portable, passive hand exoskeleton for stroke rehabilitation," IEEE Transactions on Neural Systems and Rehabilitation Engineering, vol. 19, no. 4, pp. 391-399, 2011.

[18] M. S. Cameirão, S. B. Badia, E. Duarte, and P. F. Verschure, "Virtual reality based rehabilitation speeds up functional recovery of the upper extremities after stroke: a randomized controlled pilot study in the acute phase of stroke using the Rehabilitation Gaming System," Restorative Neurology and Neuroscience, vol. 29, no. 5, pp. 287-298, 2011.

[19] M. K. Holden, T. A. Dyar, and L. Dayan-Cimadoro, "Telerehabilitation using a virtual environment improves upper extremity function in patients with stroke," IEEE Transactions on Neural Systems and Rehabilitation Engineering, vol. 15, no. 1, pp. 36-42, 2007.

[20] R. C. Loureiro, W. S. Harwin, K. Nagai, and M. Johnson, "Advances in upper limb stroke rehabilitation: a technology push," Medical \& Biological Engineering \& Computing, vol. 49, no. 10, pp. 1103-1118, 2011.

[21] S. V. Adamovich, A. Mathai, G. G. Fluet, Q. Qiu, J. Lewis, and A. S. Merians, "Design of a complex virtual reality simulation 
to train finger motion for persons with hemiparesis: a proof of concept study," Journal of NeuroEngineering and Rehabilitation, vol. 6, no. 1, p. 28, 2009.

[22] R. Colombo, F. Pisano, A. Mazzone et al., "Design strategies to improve patient motivation during robot-aided rehabilitation," Journal of NeuroEngineering and Rehabilitation, vol. 4, p. 3, 2007.

[23] C. R. Carignan and H. I. Krebs, "Telerehabilitation robotics: bright lights, big future?" Journal of Rehabilitation Research and Development, vol. 43, no. 5, pp. 695-710, 2006.

[24] P. Langhorne, F. Coupar, and A. Pollock, "Motor recovery after stroke: a systematic review," The Lancet Neurology, vol. 8, no. 8, pp. 741-754, 2009.

[25] L. Richards, C. Hanson, M. Wellborn, and A. Sethi, "Driving motor recovery after stroke," Topics in Stroke Rehabilitation, vol. 15 , no. 5, pp. 397-411, 2008.

[26] P. Langhorne, J. Bernhardt, and G. Kwakkel, "Stroke rehabilitation,” The Lancet, vol. 377, no. 9778, pp. 1693-1702, 2011.

[27] B. M. Quaney, J. He, G. Timberlake, K. Dodd, and C. Carr, "Visuomotor training improves stroke-related ipsilesional upper extremity impairments," Neurorehabilitation and Neural Repair, vol. 24, no. 1, pp. 52-61, 2010.

[28] B. I. Molier, E. H. F. van Asseldonk, H. J. Hermens, and M. J. A. Jannink, "Nature, timing, frequency and type of augmented feedback; does it influence motor relearning of the hemiparetic arm after stroke? A systematic review," Disability and Rehabilitation, vol. 32, no. 22, pp. 1799-1809, 2010.

[29] N. J. Seo, H. W. Fischer, R. A. Bogey, W. Z. Rymer, and D. G. Kamper, "Use of visual force feedback to improve digit force direction during pinch grip in persons with stroke: a pilot study," Archives of Physical Medicine and Rehabilitation, vol. 92, no. 1, pp. 24-30, 2011.

[30] K. E. Laver, S. George, S. Thomas, J. E. Deutsch, and M. Deutsch, "Virtual reality for stroke rehabilitation," The Cochrane Database of Systematic Reviews, vol. 9, Article ID CD008349, 2011.

[31] M. R. Mouawad, C. G. Doust, M. D. Max, and P. A. McNulty, "Wii-based movement therapy to promote improved upper extremity function post-stroke: a pilot study," Journal of Rehabilitation Medicine, vol. 43, no. 6, pp. 527-533, 2011.

[32] M. S. Cameirão, S. B. I. Badia, E. D. Oller, and P. F. M. J. Verschure, "Neurorehabilitation using the virtual reality based Rehabilitation Gaming System: methodology, design, psychometrics, usability and validation," Journal of Neuro Engineering and Rehabilitation, vol. 7, no. 1, p. 48, 2010.

[33] C. Vincent, I. Deaudelin, L. Robichaud et al., "Rehabilitation needs for older adults with stroke living at home: perceptions of four populations," BMC Geriatrics, vol. 7, article 20, 2007.

[34] G. Kwakkel, B. Kollen, and E. Lindeman, "Understanding the pattern of functional recovery after stroke: facts and theories," Restorative Neurology and Neuroscience, vol. 22, no. 3-5, pp. 281-299, 2004.

[35] G. Kwakkel, R. van Peppen, R. C. Wagenaar et al., "Effects of augmented exercise therapy time after stroke: a meta-analysis," Stroke, vol. 35, no. 11, pp. 2529-2536, 2004.

[36] J. W. Krakauer, "Motor learning: Its relevance to stroke recovery and neurorehabilitation," Current Opinion in Neurology, vol. 19, no. 1, pp. 84-90, 2006.

[37] B. H. Dobkin, "Training and exercise to drive poststroke recovery," Nature Clinical Practice Neurology, vol. 4, no. 2, pp. 76-85, 2008.

[38] U. Sabatini, D. Toni, P. Pantano et al., "Motor recovery after early brain damage: a case of brain plasticity," Stroke, vol. 25, no. 2, pp. 514-517, 1994.
[39] R. J. Seitz, C. M. Bütefisch, R. Kleiser, and V. Hömberg, "Reorganisation of cerebral circuits in human ischemic brain disease," Restorative Neurology and Neuroscience, vol. 22, no. 3-5, pp. 207-229, 2004.

[40] T. H. Murphy and D. Corbett, "Plasticity during stroke recovery: from synapse to behaviour," Nature Reviews Neuroscience, vol. 10, no. 12, pp. 861-872, 2009.

[41] S. C. Cramer, "Repairing the human brain after stroke: I. Mechanisms of spontaneous recovery," Annals of Neurology, vol. 63, no. 3, pp. 272-287, 2008.

[42] C. M. Bütefisch, R. Kleiser, and R. J. Seitz, "Post-lesional cerebral reorganisation: evidence from functional neuroimaging and transcranial magnetic stimulation," Journal of Physiology Paris, vol. 99, no. 4-6, pp. 437-454, 2006.

[43] S. Sonoda, E. Saitoh, S. Nagai, M. Kawakita, and Y. Kanada, "Full-time integrated treatment program, a new system for stroke rehabilitation in Japan: comparison with conventional rehabilitation," American Journal of Physical Medicine and Rehabilitation, vol. 83, no. 2, pp. 88-93, 2004.

[44] G. Rizzolatti and L. Craighero, "The mirror-neuron system," Annual Review of Neuroscience, vol. 27, pp. 169-192, 2004.

[45] G. Rizzolatti, M. Fabbri-Destro, and L. Cattaneo, "Mirror neurons and their clinical relevance," Nature Clinical Practice Neurology, vol. 5, no. 1, pp. 24-34, 2009.

[46] V. Gallese, L. Fadiga, L. Fogassi, and G. Rizzolatti, "Action recognition in the premotor cortex," Brain, vol. 119, no. 2, pp. 593-609, 1996.

[47] G. di Pellegrino, L. Fadiga, L. Fogassi, V. Gallese, and G. Rizzolatti, "Understanding motor events: a neurophysiological study," Experimental Brain Research, vol. 91, no. 1, pp. 176180, 1992.

[48] D. Ertelt, S. Small, A. Solodkin et al., "Action observation has a positive impact on rehabilitation of motor deficits after stroke," NeuroImage, vol. 36, supplement 2, pp. T164-T173, 2007.

[49] M. E. Michielsen, R. W. Selles, J. N van der Geest et al., "Motor recovery and cortical reorganization after mirror therapy in chronic stroke patients: a phase II randomized controlled trial," Neurorehabilitation and Neural Repair, vol. 25, no. 3, pp. 223-233, 2011.

[50] S. V. Adamovich, K. August, A. Merians, and E. Tunik, "A virtual reality-based system integrated with fmri to study neural mechanisms of action observation-execution: a proof of concept study," Restorative Neurology and Neuroscience, vol. 27, no. 3, pp. 209-223, 2009.

[51] A. S. Merians, E. Tunik, G. G. Fluet, Q. Qiu, and V. S. Adamovich, "Innovative approaches to the rehabilitation of upper extremity hemiparesis using virtual environments," European Journal of Physical and Rehabilitation Medicine, vol. 45, no. 1, pp. 123-133, 2009.

[52] A. R. Fugl Meyer, L. Jaasko, and I. Leyman, "The post stroke hemiplegic patient. I. A method for evaluation of physical performance," Scandinavian Journal of Rehabilitation Medicine, vol. 7, no. 1, pp. 13-31, 1975.

[53] S. Barreca, C. Gowland, P. Stratford et al., "Development of the chedoke arm and hand activity inventory: theoretical constructs, item generation, and selection," Topics in Stroke Rehabilitation, vol. 11, no. 4, pp. 31-42, 2004.

[54] S. M. Waller and J. Whitall, "Bilateral arm training: why and who benefits?” NeuroRehabilitation, vol. 23, no. 1, pp. 29-41, 2008.

[55] G. N. Lewis and W. D. Byblow, "Neurophysiological and behavioral adaptations to a bilateral training intervention in 
individuals following stroke," Clinical Rehabilitation, vol. 18, no. 1, pp. 48-59, 2004.

[56] J. H. Cauraugh, S. A. Coombes, N. Lodha, S. K. Naik, and J. J. Summers, "Upper extremity improvements in chronic stroke: coupled bilateral load training," Restorative Neurology and Neuroscience, vol. 27, no. 1, pp. 17-25, 2009.

[57] A. R. Luft, S. McCombe-Waller, J. Whitall et al., "Repetitive bilateral arm training and motor cortex activation in chronic stroke: a randomized controlled trial," Journal of the American Medical Association, vol. 292, no. 15, pp. 1853-1861, 2004.

[58] M. Wiesendanger and D. J. Serrien, "The quest to understand bimanual coordination," Progress in Brain Research, vol. 143, pp. 491-505, 2004.

[59] D. K. Rose and C. J. Winstein, "Bimanual training after stroke: are two hands better than one?" Topics in Stroke Rehabilitation, vol. 11, no. 4, pp. 20-30, 2004.

[60] C. Y. Wu, C. A. Trombly, K. C. Lin, and L. Tickle-Degnen, "A kinematic study of contextual effects on reaching performance in persons with and without stroke: influences of object availability," Archives of Physical Medicine and Rehabilitation, vol. 81, no. 1, pp. 95-101, 2000.

[61] G. Freytag and E. J. MacEwan, Freytag's Technique of the Drama: An Exposition of Dramatic Composition and Art, SC Griggs, Chicago, Ill, USA, 1894.

[62] L. von Ahn and L. Dabbish, "Designing games with a purpose," Communications of the ACM, vol. 51, no. 8 , pp. 58-67, 2008.

[63] R. M. Yerkes and J. D. Dodson, "The relation of strength of stimulus to rapidity of habit-formation," Journal of Comparative Neurology and Psychology, vol. 18, no. 5, pp. 459-482, 1908.

[64] E. Duffy, Activation and Behavior, Wiley, New York, NY, USA, 1962.

[65] R. B. Malmo, "Activation: a neuropsychological dimension," Psychological Review, vol. 66, no. 6, pp. 367-386, 1959.

[66] M. Csikszentmihalyi, Beyond Boredom and Anxiety: Experiencing Flow in Work and Play, Behavioral Science Series, chapter 14, Jossey-Bass, San Francisco, Calif, USA, 1st edition, 1975.

[67] R. H. Fortinsky, C. V. Granger, and G. B. Seltzer, "The use of functional assessment in understanding home care needs," Medical Care, vol. 19, no. 5, pp. 489-497, 1981.

[68] Z. Mathews, S. B. I. Badia, and P. F. M. J. Verschure, "A novel brain-based approach for multi-modal multi-target tracking in a mixed reality space," in Proceedings of the 4th International Conference and Workshop on Virtual Reality (INTUITION '07), IEEE, Athens, Greece, October 2007.

[69] C. C. Tsao and M. M. Mirbagheri, "Upper limb impairments associated with spasticity in neurological disorders," Journal of NeuroEngineering and Rehabilitation, vol. 4, no. 1, p. 5, 2007.

[70] K. L. Beissner, J. E. Collins, and H. Holmes, "Muscle force and range of motion as predictors of function in older adults," Physical Therapy, vol. 80, no. 6, pp. 556-563, 2000.

[71] T. W. Malone, Heuristics for Designing Enjoyable User Interfaces: Lessons from Computer Games, Ablex Publishing, Norwood, NJ, USA, 1984.

[72] A. Caria, C. Weber, D. Brötz et al., "Chronic stroke recovery after combined BCI training and physiotherapy: a case report," Psychophysiology, vol. 48, no. 4, pp. 578-582, 2010.

[73] A. C. Lo, P. D. Guarino, L. G. Richards et al., "Robot-assisted therapy for long-term upper-limb impairment after stroke," The New England Journal of Medicine, vol. 362, no. 19, pp. 1772-1783, 2010.

[74] X. L. Hu, K. Y. Tong, R. Song, X. J. Zheng, and W. W. F. Leung, "A comparison between electromyography-driven robot and passive motion device on wrist rehabilitation for chronic stroke," Neurorehabilitation and Neural Repair, vol. 23, no. 8, pp. 837-846, 2009.

[75] A. Montagner, A. Frisoli, L. Borelli et al., "A pilot clinical study on robotic assissted rehabilitation in VR with an arm exoskeleton device," in Proceedings of the Virtual Rehabilitation, pp. 5764, Venice, Italy, September 2007.

[76] M. Guidali, A. Duschau-Wicke, S. Broggi, V. KlamrothMarganska, T. Nef, and R. Riener, "A robotic system to train activities of daily living in a virtual environment," Medical \& Biological Engineering \& Computing, vol. 49, no. 10, pp. 12131223, 2011.

[77] R. J. Sanchez, J. Liu, S. Rao et al., "Automating arm movement training following severe stroke: functional exercises with quantitative feedback in a gravity-reduced environment," IEEE Transactions on Neural Systems and Rehabilitation Engineering, vol. 14, no. 3, pp. 378-389, 2006. 


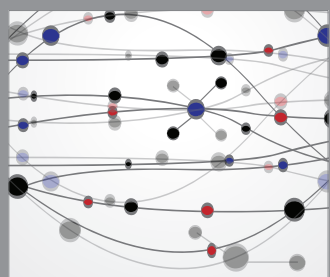

The Scientific World Journal
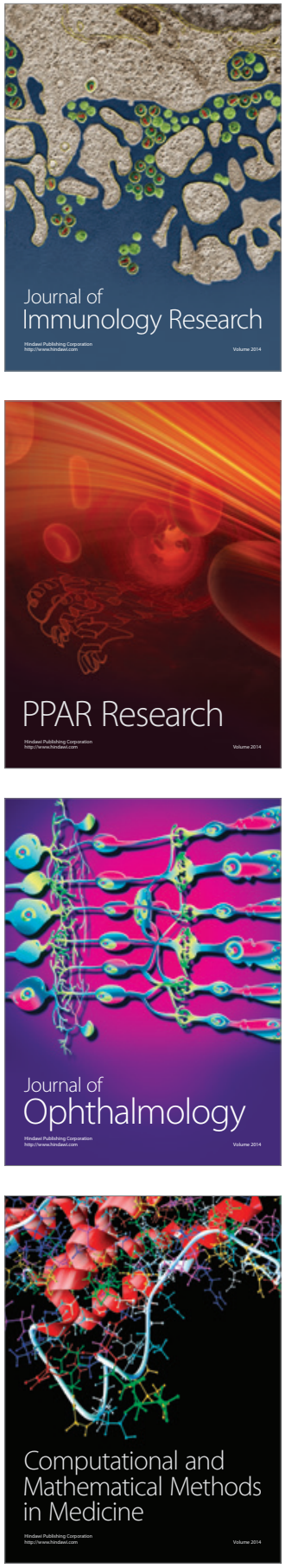

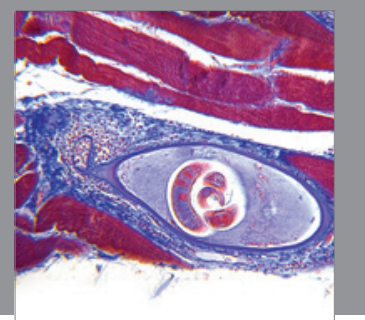

Gastroenterology

Research and Practice
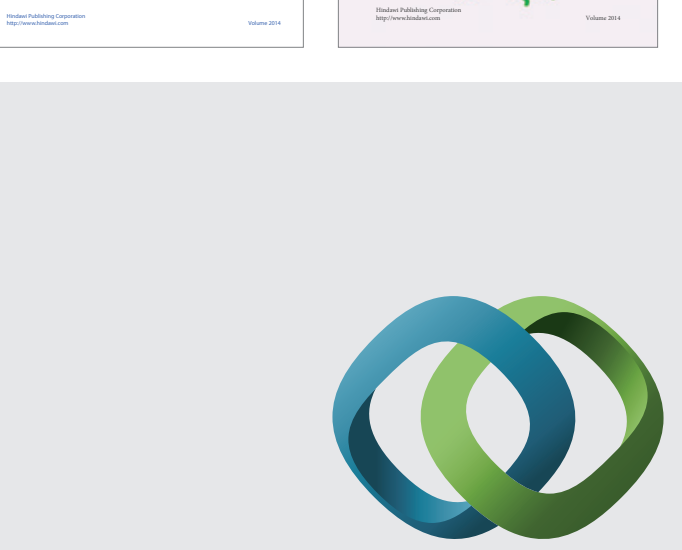

\section{Hindawi}

Submit your manuscripts at

http://www.hindawi.com
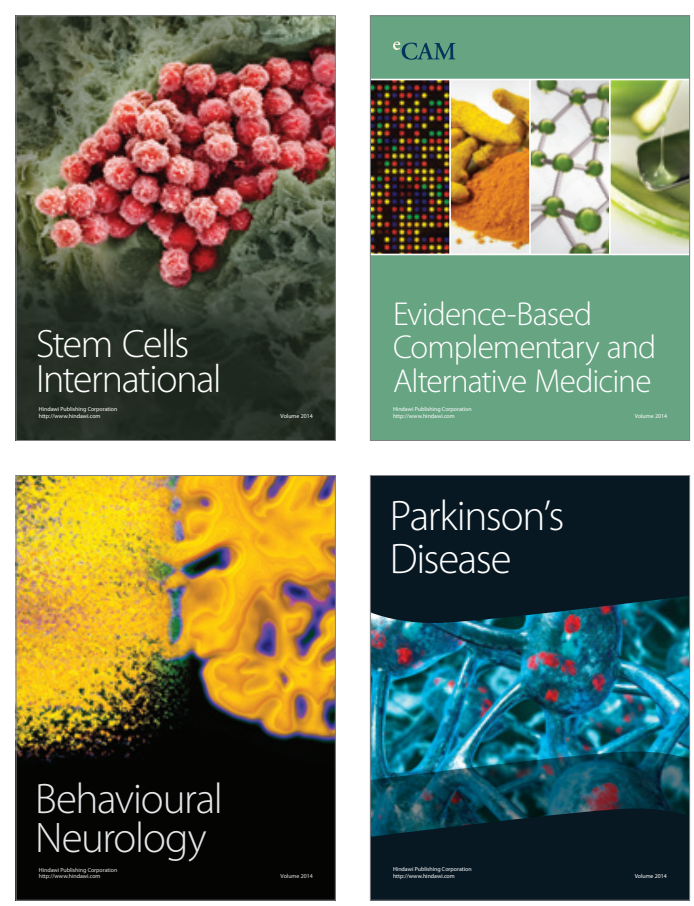

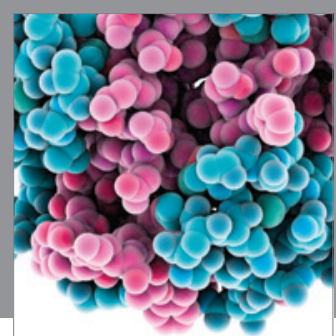

Journal of
Diabetes Research

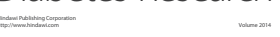

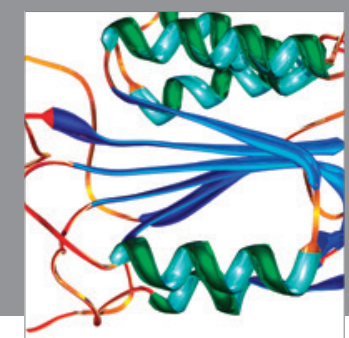

Disease Markers
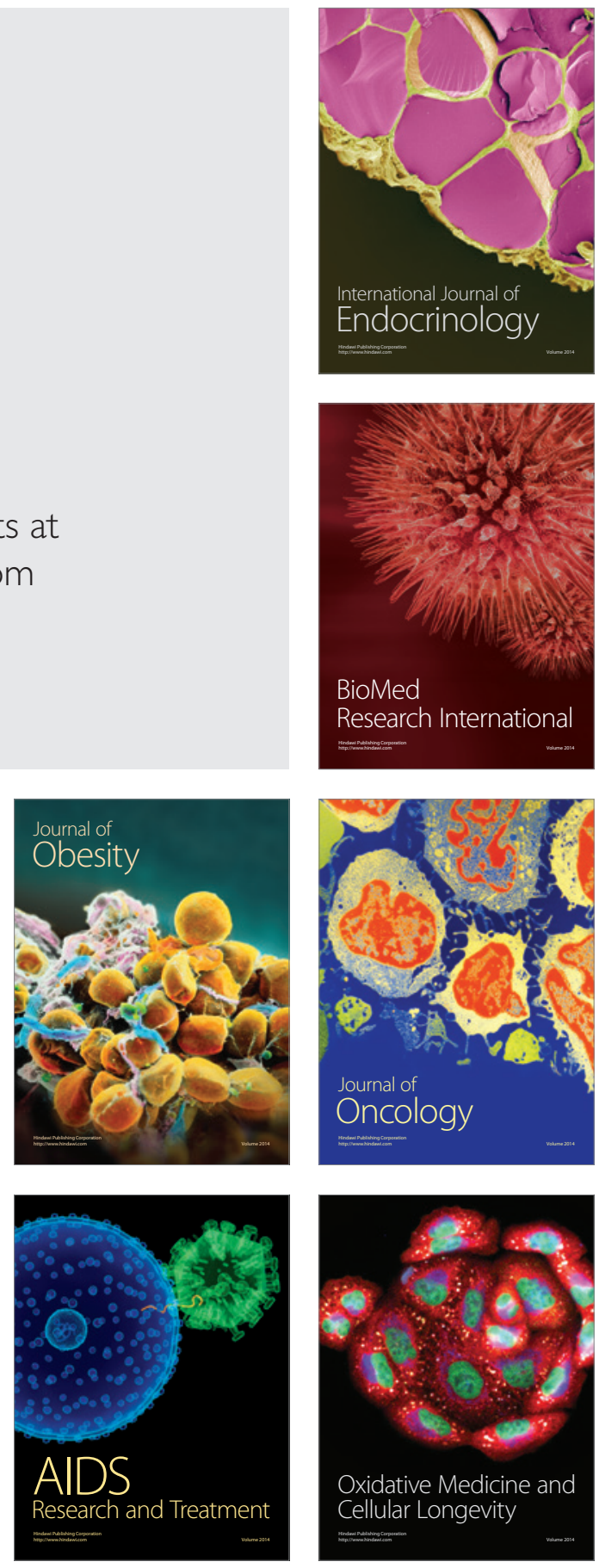